\title{
ACESSO DA POPULAÇÃO MASCULINA AOS SERVIÇOS PÚBLICOS DE SAÚDE: QUANDO OS HOMENS PROCURAM ASSISTÊNCIA?
}

\author{
Arleusson Ricarte de OLIVEIRA ${ }^{1}$ \\ Mauricéia de Albuquerque MELO ${ }^{5}$ \\ Ana Elisa Pereira CHAVES ${ }^{3}$ \\ Francisco Tobias Moreira RODRIGUES ${ }^{4}$ \\ Déborah Karollyne Ribeiro RAMOS ${ }^{5}$
}

\footnotetext{
${ }^{1}$ Enfermeiro. Mestre em Enfermagem pela Universidade Federal da Paraíba. Docente do Curso de Enfermagem da Universidade Federal de Campina Grande (UFCG) e da Faculdade de Ciências Médicas de Campina Grande (FCM). Email: ricarte27@ hotmail.com.br

${ }^{2}$ Melo. Enfermeira. Hospital Universitário Alcides Carneiro - HUAC. Email: mauryenf@gmail.com

${ }^{3}$ Enfermeira. Mestre em Enfermagem pela Universidade Federal da Paraíba. Docente do Curso de Enfermagem da Universidade Federal de Campina Grande (UFCG). E-mail: aepchaves @ hotmail.com

4 Acadêmico de Enfermagem. Universidade Estadual da Paraíba. Campina Grande, PB. Email: tobiasrodrigues@ hotmail.com

${ }^{5}$. Enfermeira. Mestre em Enfermagem pela Universidade Federal do Rio Grande do Norte. Docente do Curso de Graduação em Enfermagem da Faculdade de Ciências Médicas de Campina Grande. E-mail: deborah.ribeiro.ramos@gmail.com
}

Recebido em: 30/05/2014 - Aprovado em: 18/09/2014 - Disponibilizado em: 15/12/2014

\begin{abstract}
Resumo
Este estudo objetivou investigar os motivos que levam os homens a buscar os serviços públicos de saúde no município de Orobó-PE. Trata-se de pesquisa qualitativa com enfoque exploratório e descritivo, realizada com 27 homens escolhidos de forma aleatória, cujo corpus originou-se da transcrição de entrevistas semiestruturadas. $\mathrm{O}$ material empírico foi analisado seguindo orientações da Técnica do Discurso do Sujeito Coletivo. Os motivos que levaram os sujeitos da pesquisa a procurar os serviços de saúde foram: caso de doença instalada; exigência do local de trabalho; incentivo de pessoas próximas. Os achados apontam para fragilidades no acesso da população masculina aos serviços de saúde no município estudado. Urge a necessidade de estabelecer medidas para facilitar o acesso dos homens aos serviços de saúde, sobretudo, na atenção primária, de modo a viabilizar ações de promoção da saúde, que possibilite essa população compreender o seu processo saúde-doença, evitando assim possíveis agravos em sua saúde.
\end{abstract}

Palavras-chave: Saúde pública. Saúde do Homem. Procura por serviços de saúde. Acesso. Serviços de Saúde.

\section{Introdução}

Os agravos à saúde do homem constituem na atualidade um problema de saúde pública. Segundo estatísticas do Ministério da Saúde, 68\% da mortalidade se concentra na população masculina, na faixa etária de 20 a 59 anos. Considerando os óbitos ocorridos no ano de 2005, verificou-se que a cada três adultos que morrem no Brasil, dois são homens. Além disso, embora a expectativa de vida dos homens tenha aumentado de 63,20 para 68,92 anos de 1991 para 2007, ela ainda se mantém 7,6 anos abaixo da média das mulheres (IBGE, 2010). 
A incidência de morbimortalidade da população masculina no Brasil tem como causas mais frequentes as doenças do aparelho circulatório, seguidas por acidentes de trânsito e violência. A taxa de mortalidade por homicídios entre homens é 12 vezes maior que a feminina, chegando a 15 vezes entre 20 e 29 anos (BRASIL, 2008). Destacam-se ainda os transtornos mentais, a Síndrome da Imunodeficiência Adquirida (AIDS), o alcoolismo, que constituem fatores relevantes ao adoecimento do homem (ABREU; CESAR; FRANCA, 2009).

Esses agravantes podem estar relacionados a maior vulnerabilidade que os homens apresentam no que diz respeito às diferenças biológicas, atitudes comportamentais, como estilo de vida, percepção de saúde e a formação cultural. Todos esses fatores se relacionam diretamente com a construção de gênero internalizada pelo homem que sofre fortes influências do meio social (ABREU; CESAR; FRANCA, 2009).

Estudos demonstram que os homens procuram com menos frequência os serviços de saúde. Quando procuram, estão com estado de doença grave, em que se faz necessária hospitalização, a exemplo de lesões graves, envenenamentos, transtornos mentais, levando mais tempo de internamento, às vezes com um comprometimento maior da saúde (COUTO et al., 2010).
Frente ao impacto causado pela morbimortalidade na população masculina, o Ministério da Saúde, criou em 2008 a Política Nacional de Atenção Integral à Saúde do Homem (PNAISH) destacando que, mesmo frente aos diferenciais de morbimortalidade já conhecidos, os homens acabam por recorrer menos aos atendimentos em serviços de saúde (BRASIL, 2008). Estes aspectos podem comprometer um dos atributos essenciais da atenção primária em saúde, que é o acesso (STARFIELD, 2002).

A PNAISH, portanto, além de evidenciar os principais fatores de morbimortalidade, explicita o reconhecimento de determinantes sociais que resultam na vulnerabilidade da população masculina aos agravos à saúde, considerando que representações sociais sobre a masculinidade vigente comprometem o acesso à atenção integral, bem como repercutem de modo crítico na vulnerabilidade dessa população a situações de violência e de risco para a saúde (BRASIL, 2008).

A Atenção Básica vem ocupando cada vez mais centralidade no SUS, com a proposta de constituir-se como ordenadora das redes de atenção à saúde e como eixo estruturante de muitos programas e projetos no âmbito federal. O reconhecimento nacional e internacional que o SUS tem conquistado com a Atenção Básica justifica-se não só pela 
rapidez e escala da sua expansão de cobertura, como também pelos resultados associados ao modelo de organização deste nível de atenção:

a Estratégia Saúde da Família (BRASIL, 2011).

Apesar do acesso aos serviços de saúde ter sido um dos eixos discutidos nas Conferências de Saúde ocorridas em todo o País, e mesmo frente aos avanços da atenção básica como porta de entrada preferencial dos usuários do SUS, há um número insuficiente de estudos envolvendo essa temática, considerando o público masculino. No sentido de aprofundar essa questão é importante dar voz aos próprios homens para melhor compreender os motivos que envolvem o seu acesso aos serviços de saúde e compreender sobre os modos de enfrentamento do processo de saúde/doença desses indivíduos.

Este estudo objetivou investigar os motivos que levam os homens a buscar os serviços públicos de saúde no município de Orobó-PE.

\section{Método}

Trata-se de uma pesquisa exploratória com abordagem qualitativa realizada no município de Orobó/PE. A cidade em questão está localizada na mesorregião Agreste do Estado de Pernambuco, ocupando uma área de $125,7 \mathrm{Km}^{2}$, distando aproximadamente 127,4 km da capital do Estado. Tem uma população de 22.865 habitantes, dos quais 11.058 são constituídos de homens e 11.807 , de mulheres (IBGE, 2010). Os serviços de saúde compõem uma rede de natureza pública, constituída por um hospital, dois centros de saúde e dez Unidades de Saúde da Família.

Participaram desta pesquisa 27 sujeitos escolhidos de forma aleatória e residentes em área adscrita de uma unidade de saúde da família localizada no município. Os critérios de inclusão foram: residir em área adscrita à unidade de saúde da família, ter entre 20 e 59 anos, faixa etária considerada pelo Ministério da Saúde como prioritária para o desenvolvimento da PNAISH (BRASIL, 2008).

A coleta das informações ocorreu por meio de entrevistas semiestruturadas que foram previamente agendadas com os participantes e realizadas de forma individual. As entrevistas foram gravadas, transcritas integralmente e utilizadas nesse estudo após consentimento dos participantes. O local de realização das entrevistas foi um espaço físico disponibilizado pelo município. As informações foram coletadas durante o mês de abril de 2011, utilizando-se como instrumento um questionário composto de duas perguntas norteadoras pertinentes ao objetivo proposto. 
Com as informações obtidas na transcrição das entrevistas, foi realizada a análise, qualitativamente, por meio da Técnica do Discurso do Sujeito Coletivo (DSC) (LEFEVRÉ; LEFEVRÉ; TEIXEIRA, 2000). A operacionalização desta técnica ocorreu em três etapas: na primeira etapa selecionaram-se as expressões-chave a partir do discurso de cada participante do estudo, ou seja, dos segmentos contínuos ou descontínuos de discurso que revelam o foco principal do seu conteúdo; a segunda etapa caracterizou-se pela identificação da idéia central de cada uma das expressões-chave. Esse momento se constituiu na síntese do conteúdo das referidas expressões; na terceira etapa, foram reunidas as expressões-chave referentes às ideias centrais, em um discurso síntese, que retrata o Discurso do Sujeito Coletivo (DSC).

A pesquisa obedeceu às normas da Resolução Nº 196/96 do Conselho Nacional de Saúde (CNS), sendo aprovada pelo Comitê de Ética em Pesquisa (CEP) do Centro de Ensino Superior e Desenvolvimento- CESED, tendo como número do protocolo de aprovação $\mathrm{n}^{0}$ 0001.0.405.000-11.

\section{Resultados e discussão}

O modo como se organiza os serviços de saúde tem gerado sentimentos contraditórios apontando aspectos relevantes que podem contribuir para reflexão constante do processo saúde-doença da população masculina na garantia do acesso aos serviços de saúde.

O Quadro 1 mostra três ideias centrais do Discurso do Sujeito Coletivo relacionadas aos motivos que levam os sujeitos da pesquisa a procurar dos serviços de saúde.

Quadro 1: Descrição do Discurso do Sujeito Coletivo em resposta à questão 1. Orobó/PE, abril de 2011.

Questão 1 - Refira os motivos que levam o Sr. a procurar os serviços de saúde?

IDEIA CENTRAL 1

Em caso de doença instalada

(Discurso do sujeito coletivo )

Só vou quando estou doente. / Porque não enxergo muito direito né .../ ... preciso usar um óculos agora, mas só procuro quando realmente tô doente. /Só procurei quando tava iniciando depressão. / Só quando o organismo pede é que eu procuro./ Eu sofri um acidente três meses atrás e o organismo deixou algumas sequelas aí eu tô procurando, mas antes aí eu não procurava.

/ Já procurei o médico mas pra dor na coluna, procurei, passou remédio, passou AAS. / Eu só procuro o médico geralmente quando tô com um problema de saúde. 


\section{IDEIA CENTRAL 2}

Por exigência do local de trabalho

\section{(Discurso do sujeito coletivo )}

Qualquer canto aqui para trabalhar fixado eles exigem que faça exames para saber como está a pessoal Quem trabalha em empresa sempre tem acesso mais fácil porque a empresa encaminha de seis em seis meses ou de ano em ano e aí faz. / Eu recordo que fui pela empresa, fiz vinte e três exames e precisei repetir tudo de volta pra poder sair da empresa. / Eu trabalhei em limpeza e todo ano faz um tipo de checape. I Eu fui tem muito tempo, quando eu trabalhava.

\section{IDEIA CENTRAL 3}

Por incentivo de pessoas próximas

\section{(Discurso do sujeito coletivo )}

A gente aqui vai ao médico também por incentivo dos amigos porque eles ficam conversando com os outros. / Eu ontem fui ao médico porque minha mulher me mandou, estava com uma dor que me entronchava todo.../ A mulher é que parou o carro para eu ir, se não, sabe quantas vezes eu iria lá? Nenhuma... / Porque a gente fica conversando com o outro sobre o que tá sentindo.... / Eu fui acompanhar minha mãe e aí não custa nada ver minha pressão / Fui praticamente obrigado pela mulher pela lei do a pulso, porque se fosse pra eu ir só! / Às vezes a mulher diz: porque não vai ao médico, na enfermeira ver a pressão? / Porque o cara tá doente e não quer ir no médico aí o outro já dar uma força!

$\mathrm{Na}$ primeira ideia central, os discursos expressam a representação social de uma saúde voltada para o modelo biomédico, curativista, hospitalocêntrico e individual, ainda presente no cotidiano dos serviços, contrapondo ao modelo de vigilância em saúde, que valoriza a promoção da saúde e prevenção de doenças. Este fato constitui uma visão medicalizadora e biologizante da saúde, com uma concepção de indivíduo como organismo biológico e não integral (VASCONCELOS; VIANA; SANTOS, 2007).

Pesquisa realizada com população masculina sobre o acesso aos serviços de saúde no Rio de Janeiro, no ano de 1994, constatou que a maioria dos homens só procura serviços de saúde em caso de doença, quando necessitam buscar ajuda para tratar um problema que acomete a sua saúde e/ou quando a dor se torna insuportável, quando há uma impossibilidade de trabalhar, às vezes apelando para medidas alternativas como tomar medicação por conta própria, tomar chás ou procurar farmácias (GOMES; NASCIMENTO; ARAÚJO, 2007). Os discursos dos entrevistados demonstram que ser homem é ser forte, exigindo transparecer 
aspecto de fortaleza mesmo que estes estejam em sofrimento físico ou psíquico.

Estudos qualitativos realizados, em duas Unidades de Saúde da Família de São Paulo/SP no ano de 2008, e em 12 Unidades de Saúde da Família de Recife/PE, em 2005, reforçam que o uso dos serviços de saúde pelos homens divergem em relação às mulheres, pois concentram-se na assistência à patologias, acidentes ou lesões, problemas odontológicos e uso de farmácias. Estes agravos são de caráter emergencial uma vez que o problema já está instalado. Um dos grandes obstáculos à promoção da saúde dos homens, discutido no processo de construção da PNAISH é a centralidade da ideia de invulnerabilidade, ou seja, o homem como ser forte, na construção de masculinidade hegemônica (FIGUEIREDO, 2005; SCHRAIBER; GOMES; COUTO, 2005).

$\mathrm{Na}$ segunda ideia central observa-se através dos discursos que o contato dos homens entrevistados com os serviços de saúde acontece por exigência do local de trabalho. Este aspecto pode estar associado ao fato de que as empresas exigem periodicamente do trabalhador a realização de exames, principalmente nos momentos de admissão e/ou demissão.

Por outro lado, os discursos proporcionam uma reflexão acerca dos motivos que levam os homens a não procurarem os serviços públicos de saúde, já que o ideal da saúde pública é a ênfase na promoção da saúde e prevenção de doenças, além da maior compreensão dos indivíduos em relação ao processo saúde-doença. A este respeito, o trabalho configura-se como uma das dificuldades referidas por homens para justificar a baixa adesão aos serviços de saúde, principalmente devido à incompatibilidade de horários entre os serviços e as empresas empregadoras (COUTO et al., 2010).

A terceira ideia central retrata que a procura dos serviços de saúde pela população masculina acontece por incentivo de pessoas próximas, a exemplo de mãe, esposa e amigos. Os depoimentos mostram que os homens não procuram os serviços de saúde por iniciativa própria, o que constitui um fator negativo, pois pode repercutir em agravos no seu estado de saúde, muitas vezes levando-os a procurar os serviços de forma tardia e em casos de doença já instalada, comprometendo assim o seu estado de saúde. Os homens têm dificuldade para procurar serviços de saúde necessitando quase sempre do incentivo de pessoas próximas, para um posicionamento frente a um problema de saúde (GOMES; NASCIMENTO; ARAÚJO, 2007).

A demanda da população masculina nos serviços de saúde é muito pequena, considerando a real necessidade desse 
público, bem como a construção de uma masculinidade construída historicamente e sócio-culturalmente (MACHADO, 2008). As explicações para a menor procura dos serviços de saúde, por parte dos homens, podem estar relacionadas ao desempenho do papel social do homem, com vistas para uma afirmação masculina que atrela o cuidar ao gênero feminino. Entende-se que a adoção de diferentes estilos de vida, reflete na utilização dos serviços de saúde, com tendência de maior procura por parte da população feminina (ABREU; CESAR; FRANCA, 2009; GOMES; NASCIMENTO; ARAÚJO, 2007).

Entre os preconceitos mais comuns atribuídos ao gênero masculino, está o fato dele ser visto como forte a partir da dimensão emocional e física, o que implica de não ser assistido e de não se cuidar. Outro aspecto é o preconceito masculino em relação ao manuseio de suas partes íntimas, visto como violação à sua condição de heterossexual. Ressalta-se ainda que a subjetividade masculina baseada na força, no domínio e mesmo no machismo não é construída sozinha já que o homem nasce e cresce num "caldo cultural" que o empurra para esse papel. Este preconceito se reflete na sociedade como um todo, nas formulações das políticas públicas e também pode ser sentido em relação aos profissionais de saúde, chefes, patrões, colegas de trabalho e mesmo parceiras que estranham quando um homem procura de forma preventiva um serviço de saúde (BRAZ, 2005).

Ao investigarmos as razões que contribuem para que os sujeitos não busquem (ou evitem) acessar os serviços de saúde, o DSC nos apresenta quatro ideais centrais: medo de descobrir doenças; rejeição da possibilidade de adoecer; ausência de informações em saúde; dificuldades estruturais e operacionais dos serviços. Vejamos o quadro seguinte:

\section{CONSIDERAÇÕES FINAIS}

Dada a importância da Política Nacional de Atenção Integral à Saúde do Homem no cenário nacional, somada a potencialidade dessa política como estruturante no SUS para ser desenvolvida no âmbito da atenção primária à saúde, merece destaque o acesso da população masculina aos serviços de saúde, mais especificamente os motivos que favorecem e/ou dificultam essa acessibilidade na perspectiva de ampliar os cuidados em saúde dessa população.

As barreiras culturais constituem um dos principais fatores que interferem no acesso dos homens aos serviços de saúde. Os estereótipos de gênero ainda presentes na sociedade atual trazem impacto negativo para 
a qualidade de vida e saúde desse grupo populacional.

Vale destacar que propostas de mudanças de paradigmas em relação à concepção de masculinidade dependem do desenvolvimento de ações pela equipe de saúde, e de políticas intersetoriais que transcendam em muito os serviços de saúde propriamente ditos, visando o despertar da população masculina para os cuidados com sua saúde, assim, evitando a morbimortalidade.

As barreiras institucionais, refletidas nas dificuldades estruturais e operacionais dos serviços, impedem a busca dos homens pelo cuidado em saúde, uma vez que os serviços não estão preparados para receber essa população e adaptados para o desenvolvimento da Política Nacional de Atenção Integral à Saúde do Homem, com ações que priorizem a promoção da saúde e prevenção de doenças.

É fundamental o estabelecimento de medidas que favoreçam e estimulem o acesso da população masculina aos serviços, em especial, na atenção básica, de modo a viabilizar ações de promoção à saúde e prevenção de doenças que permitam a detecção precoce o mais rápido possível dos agravos à saúde, evitando o aparecimento de complicações potencialmente preveníveis.
Espera-se que este estudo contribua para ampliar o acervo bibliográfico acerca da temática envolvendo a saúde do homem, contribuindo com informações para que os profissionais dos serviços de saúde possam constituir suas ações no atendimento a população masculina de modo a fomentar e manter a inclusão efetiva desses indivíduos na rede de atenção à saúde. Além disso, esperase que de posse de tais informações os profissionais direcionem de acordo com as necessidades expostas pela população, de modo que estes possam ser beneficiados com o acesso aos serviços e atendidos em suas necessidades.

\section{REFERÊNCIAS}

ABREU, D.M.X.; CESAR, C.C.; FRANCA, E.B. Diferenciais entre homens e mulheres na mortalidade evitável no Brasil (1983-2005).

Cad. Saúde Pública. v. 25, n. 12, p. 2672-

2682, 2009. Disponível em: < http://www.scielo.br/scielo.php?pid=S0102$\underline{311 X 2009001200014 \& \text { script=sci_abstract\&tl }}$ $\underline{n g}=\mathrm{pt}>$ Acesso em: 30 mai. 2014.

BRASIL. Ministério da Saúde. Política Nacional de Atenção Integral à Saúde do Homem: Princípios e Diretrizes. Brasília (DF): Ministério da Saúde, 2008. Disponível em: < 
http://dtr2001.saude.gov.br/sas/PORTARIAS/

Port2008/PT-09-CONS.pdf> Acesso em: 30 mai. 2014.

BRASIL. Ministério da Saúde. Plano

Nacional de Saúde: PNS 2012-2015. Brasília

(DF ): Ministério da Saúde, 2011. Disponível em: <

http://conselho.saude.gov.br/biblioteca/Relato rios/plano_nacional_saude_2012_2015.pdf>

Acesso em: 30 mai. 2014.

BRAZ, M. A construção da subjetividade masculina e seu impacto sobre a saúde do homem: reflexão bioética sobre justiça distributiva. Ciênc. saúde coletiva. v. 10, n. 1, p. 97-104, 2005. Disponível em: < http://www.scielo.br/scielo.php?pid=S1413$\underline{81232005000100016 \& \text { script }=\text { sci_arttext }}>$.

Acesso em: 30 mai. 2014.

COUTO, M.I. et al. O Homem na Atenção Primária a Saúde: Discutindo (in) Visibilidade a partir da Perspectiva de Gênero. Interface Comunicação, Saúde, Educação. v. 14, n. 33, p. 257-270, 2010. Disponível em: < http://www.scielo.br/pdf/icse/v14n33/a03v14 n33.pdf>. Acesso em: 30 mai. 2014.

FIGUEIREDO, W. 2005. Assistência à saúde dos homens: um desafio para os serviços de Atenção Primária. Ciênc. saúde coletiva. v.
10, n. 1, p. 105-109, 2005.Disponível em: < http://www.scielo.br/pdf/csc/v10n1/a11v10n1 .pdf>. Acesso em: 30 mai. 2014.

GOMES, R.; NASCIMENTO, E.F.; ARAÚJO, F.C. Por que os homens buscam menos os serviços de saúde do que as mulheres? As explicações de homens com baixa escolaridade e homens com ensino superior. Cad. Saúde Pública. v. 23, n. 3, p. 565-574, 2007. Disponível em: < http://www.scielosp.org/pdf/csp/v23n3/15.pdf >. Acesso em: 30 mai. 2014.

\section{INSTITUTO BRASILEIRO DE} GEOGRAFIA E ESTATÍSTICA. Contagem da população 2010. Brasília (Brasil): Ministério do Planejamento, Orçamento e Gestão, 2010.

LEFÉVRE, F.; LEFÉVRE, A.M.C.; TEIXEIRA, J.J.V. O discurso do sujeito coletivo: uma nova abordagem metodológica em pesquisa qualitativa.

Caxias do Sul: EDUSC; 2000.

MACHADO, F.R. Grupo de Homens: repensando o Papel Masculino na Sociedade Contemporânea. Rev. Cient. de Psicologia. v. 2, n. 1, p. 1-31, 2008. Disponível em: < http://www.pesquisapsicologica.pro.br/pub02/ fabio.pdf>. Acesso em: 30 mai. 2014. 
SCHRAIBER, L.B.; GOMES, R.; COUTO,

M.T. Homens e saúde na pauta da Saúde

Coletiva. Ciênc. saúde coletiva. v. 10, n. 1, p.

7-17, 2005. Disponível em: <

http://www.scielo.br/pdf/csc/v10n1/a02v10n1

>. Acesso em: 30 mai. 2014.

STARFIELD, B. Atenção primária:

equilíbrio entre necessidades de saúde,

serviços e tecnologia. Brasília: UNESCO,

Ministério da Saúde, 2002.

VASCONCELOS, K.M.; VIANA, K.M.P.;

SANTOS, M.F.S. Pensando o método de

pesquisa em representação social. In:

RODRIGUES, M.M.P.; MENANDRO, M.M.

(Orgs.). Lógicas metodológicas: trajetos de

pesquisa em psicologia. Vitória: Gráfica

Editora. UFES/ GM, 2007. p. 39-56. 